\title{
INTERACTIVE COMPROMISE HYPERSPHERE METHOD AND ITS APPLICATIONS
}

\author{
SEbastian Sitarz ${ }^{1}$
}

\begin{abstract}
The paper focuses on multi-criteria problems. It presents the interactive compromise hypersphere method with sensitivity analysis as a decision tool in multi-objective programming problems. The method is based on finding a hypersphere (in the criteria space) which is closest to the set of chosen nondominated solutions. The proposed modifications of the compromise hypersphere method are based on using various metrics and analyzing their influence on the original method. Applications of the proposed method are presented in four multi-criteria problems: the assignment problem, the knapsack problem, the project management problem and the manufacturing problem.
\end{abstract}

Keywords. Multi-criteria problems, multiple objective linear programming, sensitivity analysis, decision making, compromise programming.

Mathematics Subject Classification. 90C29, 90B50, 49Q12.

\section{INTRODUCTION}

The aim of this paper is to present a method of ranking the nondominated solutions in multiple objective linear programming problems. The main idea of the proposed method comes from the work of Gass and Roy [14]. In their work only one metric is used to generate ranking by compromise hypersphere. We propose to use various metrics and analyze their influence on the original method. Moreover, we present the interactive version of the method to include the decision maker's preferences. The motivation to study various metrics is to show the decision maker's

Received August 13, 2009. Accepted August 2, 2012.

1 Institute of Mathematics, University of Silesia, ul. Bankowa 14, 40-007 Katowice, Poland. ssitarz@math.us.edu.pl 
risk aversion by means of the chosen metric. The presented analysis will provide us with remarks how to choose the proper metric.

The paper consists of the following sections. Section 2 presents multi-objective linear programming (MOLP) problem and description of the method by using various metrics. Section 3 gives the properties of the proposed method. Section 4 proposes a new interactive multicriteria method - the ICOH method with sensitivity analysis. Section 5 presents applications of the ICOH method. The paper is summarized in the final section 6 .

Other works considering the problem of metrics in compromise programming are also worth mentioning. In this article we consider various metrics in compromise approach, similarly to [2]. In work [2] Ballestero studies the problem of selection of the compromise programming metric in the view of risk aversion and obtains a reliable specification of the metric defining the compromise distance. Similar problem of choosing a metric (a norm) in compromise programming was considered in work [5] by Carrizosa et al. which considers so called AS norms in the ideal-point methods.

\section{COMPROMise HYPERSPHERE METHOD}

\subsection{Multi-OBJective PRoBlems}

We consider the following (MOP) multi-objective programming problem:

$$
v \max \{\mathbf{C x}: \mathbf{x} \in X\}
$$

where $X \subset \mathcal{R}^{N}$ is a feasible region, $\mathbf{C} \in \mathcal{R}^{k \times N}$ is a matrix of objective function coefficients.

We call $\mathbf{y} * \in \mathcal{R}^{k}$ a nondominated solution of problem (2.1) if there is $\mathbf{x} * \in X$ such that

$$
\mathbf{y} *=\mathbf{C x} *
$$

and there is no $\mathbf{x}^{\prime} \in X$ such that

$$
\mathrm{Cx}^{*} \leq \mathrm{Cx}^{\prime} \wedge \mathrm{Cx}^{*} \neq \mathrm{Cx}^{\prime}
$$

We obtain the MOLP (multi-objective linear programming) problem by using the following set of feasible region:

$$
X=\left\{\mathbf{x} \in \mathcal{R}^{N}: \mathbf{A x} \leq \mathbf{b}, \mathbf{x} \geq 0\right\}
$$

where $\mathbf{A} \in \mathcal{R}^{m \times N}$ is a matrix of constraint coefficients and $\mathbf{b} \in \mathcal{R}^{m}$ is a right hand side vector.

We call $\mathbf{y}^{*} \in \mathcal{R}^{k}$ a nondominated extreme solution of MOLP if $\mathbf{y}^{*}$ is an nondominated solution and $\mathbf{y}^{*}$ is an extreme point of set $Y=\mathbf{C}(X)$. 


\subsection{Description of the Method}

The method consists of three steps. The general description of the presented steps is as follows. Step 1 presents an initial problem of decision making - finding a set of nondominated solutions. This problem in MOLP was analyzed very often for example in $[30,37,38]$. In step 2 we look for a hypersphere which we call a compromise hypersphere. In this case the word compromise means a surface of compromise over the values of nondominated extreme solutions. Thus, we look for the points which are closest to this surface. It means that the point closest to this surface is the best compromise solution. In step 3 we built ranking of points by using distance to the compromise hypersphere.

The aim of the presented method is to rank the nondominated solutions of problem (2.1). In detail, the method looks as follows:

Step 1. Determine a finite set of nondominated solutions of problem (2.1) (in MOLP: nondominated extreme solutions). We will denote these nondominated solutions as: $\mathbf{y}^{1}, \mathbf{y}^{2}, \ldots, \mathbf{y}^{n}$.

Step 2. Find a hypersphere with the centre $\mathbf{y}^{0} \in \mathcal{R}^{k}$ and radius $r_{0} \in \mathcal{R}$ by solving the program

$$
\min _{\mathbf{y}^{0}, r_{0}} l_{q}^{n}\left(\left[r_{0}, \ldots, r_{0}\right],\left[l_{p}^{k}\left(\mathbf{y}^{1}, \mathbf{y}^{0}\right), \ldots, l_{p}^{k}\left(\mathbf{y}^{n}, \mathbf{y}^{0}\right)\right]\right)
$$

where

$p, q \in[1, \infty]$ are parameters

$l_{q}^{n}$ and $l_{p}^{k}$ are functions belonging to the family of metrics $l_{r}^{s}: \mathcal{R}^{s} \times R^{s} \rightarrow \mathcal{R}$ with the following form:

$$
l_{r}^{s}(\mathbf{a}, \mathbf{b})=\left\{\begin{array}{c}
\sqrt[r]{\sum_{i=1}^{s}\left|a_{i}-b_{i}\right|^{r}}, r \in[1, \infty) \\
\max _{i=1, \ldots, s}\left|a_{i}-b_{i}\right|, r=\infty .
\end{array}\right.
$$

We will denote the optimal solution of $(2.2)$ as ${\overline{\mathbf{y}^{0}}}^{p, q},{\overline{r_{0}}}^{p, q}$ and the minimal value of the cost function as $\overline{\min }(2.2)^{p, q}$. Moreover, wherever it does not cause notion misunderstanding, we will omit indices $p, q$ i.e. $\overline{\mathbf{y}^{0}}, \overline{r_{0}}$ and $\overline{\min }(2.2)$.

Step 3. Find the ranking of the points $\mathbf{y}^{1}, \mathbf{y}^{2}, \ldots, \mathbf{y}^{n}$ based on the distance from the previously found hypersphere in step (2.2), using values

$$
\left|\overline{r_{0}}-l_{p}^{k}\left(\mathbf{y}^{i}, \mathbf{y}^{0}\right)\right|
$$

We will particularly look for the point $\mathbf{y}^{i}$ closest to the hypersphere:

$$
\min _{i}\left|\overline{r_{0}}-l_{p}^{k}\left(\mathbf{y}^{i}, \mathbf{y}^{0}\right)\right|
$$

We will denote the optimal solution of (2.3) as $\bar{i}$, the optimal extreme point as $\overline{\mathbf{y}^{i}}$, and the minimal value of the cost function as $\overline{\min }(2.3)$. 


\subsection{NumericAl EXAMPLE}

Let us consider the example from the work of Gass and Roy (example 4, [14]), where the problem of MOLP has the following form:

$$
\begin{aligned}
v \max & {\left[x_{1}, x_{2}\right] } \\
x_{1}+x_{2} & \leq 8 \\
2 x_{1}+x_{2} & \leq 12 \\
x_{1}+2 x_{2} & \leq 14 \\
9 x_{1}+7 x_{2} & \leq 63 \\
-4 x_{1}+10 x_{2} & \leq 61 \\
2 x_{1}-x_{2} & \leq 8 \\
14 x_{1}+3 x_{2} & \leq 72 \\
x_{1}, x_{2} & \geq 0 .
\end{aligned}
$$

We will conduct the method described in Section 2.2.

Step 1. The nondominated extreme points have the following form:

$$
\begin{aligned}
\mathbf{y}^{1} & =(4.8,1.6), \mathbf{y}^{2}=(4.5,3.0), \mathbf{y}^{3}=(4.2,3.6), \mathbf{y}^{4}=(3.5,4.5), \mathbf{y}^{5} \\
& =(2.0,6.0), \mathbf{y}^{6}=(1.0,6.5) .
\end{aligned}
$$

Step 2. Generally, problem (2.2) is a nonconvex problem with a lot of local optima. In order to solve this problem, genetic algorithms are used. We do not discuss computational analysis of such problems in this paper, however it is an interesting issue which needs further research (a separate paper). Let us discuss the case when $p=1,2, \infty$ and $q=\infty$. The optimal values of the objective function of the problem (2.2) are as follows:

$$
\overline{\min }(2.2)^{1, \infty}=0.25, \quad \overline{\min }(2.2)^{2, \infty}=0.0516, \quad \overline{\min }(2.2)^{\infty, \infty}=0.65 .
$$

Step 3. On the basis of the distance to the previously found hypersphere we obtain rankings presented in Table 1 . Some values of the parameter $p$ generate more than one ranking, which is the consequence of the alternative optimal solutions of problem (2.2).

\section{Properties of the Compromise hypersphere Method}

We present the properties of the proposed method to highlight the modifications and improvements proposed to the original algorithm by Gass and Roy [14]. Generally, Step 1 is the same like in the original algorithm, but Steps 2 and 3 are different by opportunity of choosing the metrics. 
TABLE 1. Rankings obtained in example 5.1 for $p=1,2, \infty$ and $q=\infty$.

\begin{tabular}{|l|l|l|l|l|}
\hline \multicolumn{2}{|c|}{$p=1$} & $p=2$ & \multicolumn{2}{|c|}{$p=\infty$} \\
\hline $1 . \mathbf{y}^{1}, \mathbf{y}^{3}$ & $1 \cdot \mathbf{y}^{3}$ & $1 \cdot \mathbf{y}^{6}$ & $1 \cdot \mathbf{y}^{3}$ & $1 \cdot \mathbf{y}^{5}$ \\
$2 \cdot \mathbf{y}^{2}, \mathbf{y}^{4}, \mathbf{y}^{5}, \mathbf{y}^{6}$ & $2 \cdot \mathbf{y}^{1}, \mathbf{y}^{6}$ & $2 \cdot \mathbf{y}^{3}$ & $2 \cdot \mathbf{y}^{5}$ & $2 \cdot \mathbf{y}^{3}$ \\
& $3 \cdot \mathbf{y}^{2}, \mathbf{y}^{4}, \mathbf{y}^{5}$ & $3 \cdot \mathbf{y}^{5}$ & $3 \cdot \mathbf{y}^{6}$ & $3 \cdot \mathbf{y}^{2}$ \\
& & $4 \cdot \mathbf{y}^{4}$ & $4 \cdot \mathbf{y}^{2}$ & $4 \cdot \mathbf{y}^{6}$ \\
& & $5 \cdot \mathbf{y}^{1}$ & $5 \cdot \mathbf{y}^{1}, \mathbf{y}^{4}$ & $5 \cdot \mathbf{y}^{1}, \mathbf{y}^{4}$ \\
& & $6 \cdot \mathbf{y}^{2}$ & & \\
\hline
\end{tabular}

\subsection{Properties of Step 1}

Remark 3.1. The nondominated extreme solutions in MOLP problem may be generated with the help of some algorithms. One of them is the ADBASE method proposed by Steuer [30] - it uses the simplex tableaus analysis. Evans and Steuer in [9] propose a method by using a revised simplex method. Yu and Zeleny in [37] generate the entire set of all nondominated solutions through the set of all nondominated extreme points. Ehrgott in [7] presents the parametric programming procedure in the case of two objective functions.

Remark 3.2. It is possible to consider any finite subset of the nondominated solutions preferred by the decision maker.

\subsection{Properties of Step 2}

Remark 3.3. It is worth noticing that the idea of considering problem (2.2) comes from the general approach to the compromise methods - the compromise solutions, see for example: [34, 38, 39].

Before we present next remarks for problem (2.2), let us look at the following theorem, which is important for the interpretation of problem (2.2).

Theorem 3.4. The distance of the point $\boldsymbol{y}^{i}$ from the hypersphere $H$ with the centre in $\boldsymbol{y}^{0}$ and the radius $r_{0}$ is described with the formula:

$$
\inf \left\{l_{p}^{k}\left(\mathbf{y}^{i}, \mathbf{y}\right): \boldsymbol{y} \in H\right\}=\left|r_{0}-l_{p}^{k}\left(\mathbf{y}^{i}, \mathbf{y}^{0}\right)\right| .
$$

Proof. [1.]

We will use the fact that the metric $l_{p}^{k}$ generates the norm:

$$
\|\mathbf{z}-\mathbf{w}\|_{p}^{k}=l_{p}^{k}(\mathbf{z}, \mathbf{w}) .
$$

2. Let us take $\mathbf{y}^{*} \in H$, which lies on the line generated by $\mathbf{y}^{0}$ and $\mathbf{y}^{i}$. Thus, the points $\mathbf{y}^{*}, \mathbf{y}^{0}, \mathbf{y}^{i}$ are collinear, which means that we get the equation in the triangular condition:

$$
\left\|\mathbf{y}^{0}-\mathbf{y}^{i}\right\|_{p}^{k}=\left\|\mathbf{y}^{0}-\mathbf{y}^{*}\right\|_{p}^{k}+\left\|\mathbf{y}^{*}-\mathbf{y}^{i}\right\|_{p}^{k}
$$


or

$$
\left\|\mathbf{y}^{0}-\mathbf{y}^{*}\right\|_{p}^{k}=\left\|\mathbf{y}^{0}-\mathbf{y}^{i}\right\|_{p}^{k}+\left\|\mathbf{y}^{i}-\mathbf{y}^{*}\right\|_{p}^{k} .
$$

3. Let us consider two cases: $3 \mathrm{a}$ and $3 \mathrm{~b}$.

3a. The point $\mathbf{y}^{i}$ lies outside or on the hypersphere, that is $l_{p}^{k}\left(\mathbf{y}^{0}, \mathbf{y}^{i}\right) \geq r_{0}$, then using (3.1) we get:

$$
\left\|\mathbf{y}^{*}-\mathbf{y}^{i}\right\|_{p}^{k}=\left\|\mathbf{y}^{0}-\mathbf{y}^{i}\right\|_{p}^{k}-r_{0} .
$$

It means that

$$
l_{p}^{k}\left(\mathbf{y}^{i}, \mathbf{y}^{*}\right)=\left|r_{0}-l_{p}^{k}\left(\mathbf{y}^{i}, \mathbf{y}^{0}\right)\right|
$$

Moreover, we have

$$
\begin{aligned}
l_{p}^{k}\left(\mathbf{y}^{i}, \mathbf{y}^{0}\right) & \leq l_{p}^{k}\left(\mathbf{y}^{i}, \mathbf{y}\right)+l_{p}^{k}\left(\mathbf{y}, \mathbf{y}^{0}\right) \\
r_{0}+l_{p}^{k}\left(\mathbf{y}^{i}, \mathbf{y}^{*}\right) & \leq l_{p}^{k}\left(\mathbf{y}^{i}, \mathbf{y}\right)+r_{0} \\
l_{p}^{k}\left(\mathbf{y}^{i}, \mathbf{y}^{*}\right) & \leq l_{p}^{k}\left(\mathbf{y}^{i}, \mathbf{y}\right) .
\end{aligned}
$$

3b. The point $\mathbf{y}^{i}$ lies inside the hypersphere, that is $l_{p}^{k}\left(\mathbf{y}^{i}, \mathbf{y}^{0}\right)<r_{0}$, then using (3.2) we get:

$$
\left\|\mathbf{y}^{*}-\mathbf{y}^{i}\right\|_{p}^{k}=r_{0}-\left\|\mathbf{y}^{0}-\mathbf{y}^{i}\right\|_{p}^{k}
$$

It means that:

$$
l_{p}^{k}\left(\mathbf{y}^{i}, \mathbf{y}^{*}\right)=\left|r_{0}-l_{p}^{k}\left(\mathbf{y}^{i}, \mathbf{y}^{0}\right)\right|
$$

Moreover, we have

$$
l_{p}^{k}\left(\mathbf{y}^{i}, \mathbf{y}^{0}\right)+l_{p}^{k}\left(\mathbf{y}^{i}, \mathbf{y}^{*}\right)=r_{0}=l_{p}^{k}\left(\mathbf{y}, \mathbf{y}^{0}\right) \leq l_{p}^{k}\left(\mathbf{y}^{i}, \mathbf{y}^{0}\right)+l_{p}^{k}\left(\mathbf{y}^{i}, \mathbf{y}\right)
$$

hence

$$
l_{p}^{k}\left(\mathbf{y}^{i}, \mathbf{y}^{*}\right) \leq l_{p}^{k}\left(\mathbf{y}^{i}, \mathbf{y}\right) .
$$

4. The points $2-3$ give us the following conclusion:

$$
\inf \left\{l_{p}^{k}\left(\mathbf{y}^{i}, \mathbf{y}\right): \mathbf{y} \in H\right\}=\left|r_{0}-l_{p}^{k}\left(\mathbf{y}^{i}, \mathbf{y}^{0}\right)\right| .
$$

Remark 3.5. The interpretation of problem (2.2) looks as follows: we look for the hypersphere which minimizes the distances from all the points in the sense of the given metrics $l_{q}^{n}$ and $l_{p}^{k}$.

Remark 3.5 is based on the following facts:

The distances of the points $\mathbf{y}^{1}, \mathbf{y}^{2}, \ldots, \mathbf{y}^{n}$ from the hypersphere with the centre in $\mathbf{y}^{0}$ and the radius $r_{0}$ are described with the following formula (using Thm. 1)

$$
\left|r_{0}-l_{p}^{k}\left(\mathbf{y}^{1}, \mathbf{y}^{0}\right)\right|, \ldots,\left|r_{0}-l_{p}^{k}\left(\mathbf{y}^{n}, \mathbf{y}^{0}\right)\right| .
$$

If we want to minimize all the above values, then we get the multi-objective problem:

$$
v \min _{\mathbf{y}^{0}, r_{0}}\left|r_{0}-l_{p}^{k}\left(\mathbf{y}^{1}, \mathbf{y}^{0}\right)\right|, \ldots,\left|r_{0}-l_{p}^{k}\left(\mathbf{y}^{n}, \mathbf{y}^{0}\right)\right| \text {. }
$$


The ideal point in the criteria space of the problem $(3.3)$ is $(0, \ldots, 0) \in \mathcal{R}^{n}$. Thus, using the metric $l_{q}^{n}$ we obtain the compromise program for (3.3) in the following form:

$$
\min _{\mathbf{y}^{0}, r_{0}} l_{q}^{n}\left((0, \ldots, 0),\left(\left|r_{0}-l_{p}^{k}\left(\mathbf{y}^{1}, \mathbf{y}^{0}\right)\right|, \ldots,\left|r_{0}-l_{p}^{k}\left(\mathbf{y}^{n}, \mathbf{y}^{0}\right)\right|\right) .\right.
$$

It is obvious that (3.4) is equivalent to the problem (2.2):

$$
\min _{\mathbf{y}^{0}, r_{0}} l_{q}^{n}\left(\left(r_{0}, \ldots, r_{0}\right),\left(l_{p}^{k}\left(\mathbf{y}^{1}, \mathbf{y}^{0}\right), \ldots, l_{p}^{k}\left(\mathbf{y}^{n}, \mathbf{y}^{0}\right)\right)\right.
$$

Remark 3.6. It is worth mentioning that in special cases problem (2.2) is considered. For example Anthony et al. [1] and Butler et al. [4] analyze problem (2.2) with $p=2$ and $q=\infty$. Whereas, an approximation of problem (2.2) with $p=2$ and $q=\infty$ is considered by Gass and Roy in [14]. The quality of this approximation may be found in [15].

\subsection{Properties of Step 3}

Remark 3.7. The problem in step 3 is trivial, it means the review of $n$ numbers:

$$
\left|\overline{r_{0}}-l_{p}^{k}\left(\mathbf{y}^{i}, \mathbf{y}^{0}\right)\right| i=1,2, \ldots, n
$$

and their array from the smallest up. The minimal value relates to the compromise solution $\overline{\mathbf{y}^{i}}$. Moreover, the ranged numbers have been used in step 2.

Remark 3.8. By omitting the module in the values $\left|\overline{r_{0}}-l_{p}^{k}\left(\mathbf{y}^{i}, \mathbf{y}^{0}\right)\right|$, that is considering the values:

$$
\overline{r_{0}}-l_{p}^{k}\left(\mathbf{y}^{i}, \mathbf{y}^{0}\right)
$$

we get the following interpretation of these values:

- a negative value means that the point $\mathbf{y}^{i}$ lies outside the hypersphere;

- a positive value means that the point $\mathbf{y}^{i}$ lies inside the hypersphere;

- a value which equals zero means that the point $\mathbf{y}^{i}$ lies on the hypersphere.

\section{INTERACTIVE COMPROMISE HYPERSPHERE METHOD - ICOH METHOD}

We are going to present a new multicriteria method - the ICOH method. This method is based on the compromise hypersphere method. We modify the initial method by including the decision maker's preferences concerning the risk aversion. In our model the risk aversion is represented by choosing the metric. 


\subsection{Choice of the Parameter $p$}

Let us notice that the choice of parameter $p$ generates the shape of the hypersphere (when $p=2$ it is a $k$-dimensional ball, for special case $k=2$ we get a circle). Our aim is to place the hypersphere closest to the points $\mathbf{y}^{1}, \mathbf{y}^{2}, \ldots, \mathbf{y}^{n}$. Our modification allows us to review the parameters $p$ in order to choose the one which generates the closest hypersphere.

Let us assume that the parameters $p$ and $q$ have been given. Moreover, the optimal solution with the optimal value of the cost function has been found:

$$
\overline{\mathbf{y}}^{p, q},{\overline{r_{0}}}^{p, q} \overline{\min }(2.2)^{p, q}
$$

Together with the given parameter $q^{*}$, value $\overline{\min }(2.2)^{p, q *}$ is shown by the compromise distance of the points $\mathbf{y}^{1}, \mathbf{y}^{2}, \ldots, \mathbf{y}^{n}$ from the hypersphere depending on $p$. The natural consequence is to choose $p$ which minimizes the above value, that is:

$$
\min _{p} \overline{\min }(2.2)^{p, q *}
$$

Problem (4.1) aims to match the shape of the compromise hypersphere with the position of the given points $\mathbf{y}^{1}, \mathbf{y}^{2}, \ldots, \mathbf{y}^{n}$. By using the case of two objectives $(k=2)$ we present the illustration of this idea in Figure 1. In this figure the points lie close to the square, it means that $p$ close to $\infty$ would be the best choice.

Remark 4.1. Using problem (4.1) leads directly to the choice of optimal value of parameter $p$, without the interference of the decision maker. On the other hand, the decision maker wants to find the best compromise solution, so we generate the best compromise value of parameter $p$. Therefore, we set parameter $p$ as an optimal solution of problem (4.1), in that way we place the hypersphere closest to the nondominated solutions.

\subsection{Choice of the Parameter $q$}

In our approach the parameter $q$ represents the way of choosing the distance in the compromise programming. Thus, this parameter is important and has influence on the final solution. On the other hand we can look at the problem of choosing the parameter $q$ analogically to the subsection 4.1. In this case, the choice of the parameter $q$ is based on the following problem :

$$
\min _{q} \overline{\min }(2.2)^{p *, q}
$$

Such a method leads directly to the choice $q=\infty$, which is shown in the following proposition. 


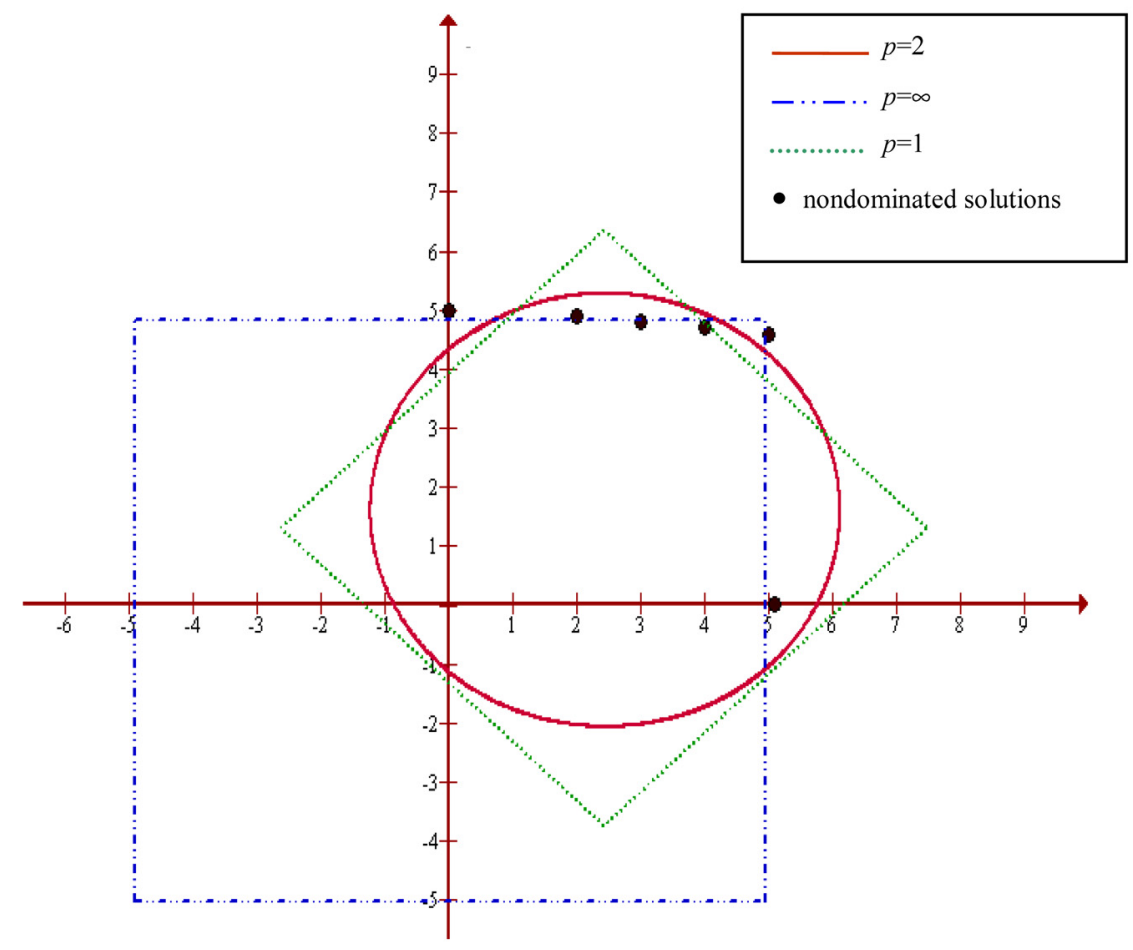

FiguRE 1 . The comparison of the compromise hyperspheres with $p=1, p=2$ and $p=\infty$ in the case of two objectives.

Proposition 4.2. If $p^{*} \in[1, \infty]$, then

$$
\min _{q} \overline{\min }(2.2)^{p *, q}=\overline{\min }(2.2)^{p *, \infty}
$$

Proof. 1. We will use the following fact for a family of metrics $l_{r}^{s}$.

For all $s \in[1, \infty]$ and $\mathbf{z}, \mathbf{w} \in \mathcal{R}^{s}$ we have:

$$
1 \leq r \leq t \leq \infty \Rightarrow, l_{r}^{s}(\mathbf{z}, \mathbf{w}) \geq l_{t}^{s}(\mathbf{z}, \mathbf{w}) .
$$

2. Let ${\overline{\mathbf{y}^{0}}}^{p * q},{\overline{r_{0}}}^{p *, q}$ be the optimal solution of $(2.2)$ with $q \in[1, \infty]$, that is:

$$
\overline{\min }(2.2)^{p *, q}=l_{q}^{n}\left(\left({\overline{r_{0}}}^{p *, q}, \ldots,{\overline{r_{0}}}^{p *, q}\right),\left(l_{p *}^{k}\left(\mathbf{y}^{1},{\overline{\mathbf{y}^{0}}}^{p *, q}\right), \ldots, l_{p *}^{k}\left(\mathbf{y}^{n},{\overline{\mathbf{y}^{0}}}^{p *, q}\right)\right)\right) .
$$

3. Using points $1-2$, we have for all $q \in[1, \infty]$ :

$$
\overline{\min }(2.2)^{p *, q} \geq l_{\infty}^{n}\left(\left({\overline{r_{0}}}^{p *, q}, \ldots,{\overline{r_{0}}}^{p *, q}\right),\left(l_{p *}^{k}\left(\mathbf{y}^{1},{\overline{\mathbf{y}^{0}}}^{p *, q}\right), \ldots, l_{p *}^{k}\left(\mathbf{y}^{n},{\overline{\mathbf{y}^{0}}}^{p *, q}\right)\right)\right) .
$$


4. It is obvious that

$$
l_{\infty}^{n}\left(\left({\overline{r_{0}}}^{p *, q}, \ldots,{\overline{r_{0}}}^{p *, q}\right),\left(l_{p *}^{k}\left(\mathbf{y}^{1},{\overline{\mathbf{y}^{0}}}^{p *, q}\right), \ldots, l_{p *}^{k}\left(\mathbf{y}^{n},{\overline{\mathbf{y}^{0}}}^{p *, q}\right)\right)\right) \geq \overline{\min }(2.2)^{p *, \infty} .
$$

5. From the points $2-4$ follows the fact that for all $q \in[1, \infty]$ we have:

$$
\overline{\min }(2.2)^{p *, q} \geq \overline{\min }(2.2)^{p *, \infty}
$$

thus

$$
\min _{q} \overline{\min }(2.2)^{p *, q}=\overline{\min }(2.2)^{p *, \infty} .
$$

\subsection{RisK AVERSION MEASURE}

Proposition 1. leads directly to the choice $q=\infty$, but assuming that $q=\infty$ at once narrows the compromise method because the choice of $q$ is an arbitrary case, similarly as in the ordinary compromise programming. The choice of the metric (the value of $q$ ) should go along with some preferences of the decision maker, which is described by Ballestero in [2] who concludes his works by sentence: "the greater risk aversion the greater $q$-metric to use. Metric $q=\infty$ corresponds to extremely high risk aversion". Thus the preferences of the decision maker can be incorporated in the presented method by choosing the parameter $q$ - representation of the risk aversion.

By using the above theory, we can formulate the following comments to setting parameter $q$ :

- $q=1$ corresponds to extremely low risk aversion;

$-q=\infty$ corresponds to extremely high risk aversion;

- $q \in(1, \infty)$ corresponds to risk aversion between low and high.

The above scale, $[1, \infty]$, can be hard to use in practice. Thus, we propose to transform interval $[1, \infty]$ to interval $[0,1]$ by a given bijection $f$, for example by $f(q)=(2 / \pi) \arctan (q-1)$ After using such a transformation $f$, the decision maker gives a value from interval $[0,1]$ to describe his risk aversion in the following way:

- $f(q)=0$ corresponds to extremely low risk aversion;

- $f(q)=1$ corresponds to extremely high risk aversion;

- $f(q) \in(0,1)$ corresponds to risk aversion between low and high.

\subsection{SEnsitivity ANALYSiS}

Sensitivity analysis in MOLP problems is considered in many works. Gal and Wolf in [10] give a survey of the literature focusing on this problem. Hansen et al. [17] and Hladik [18] present sensitivity analysis by means of a tolerance approach. Sitarz $([23,25])$ presents the standard sensitivity approach in MOLP and in [28] presents volume-based sensitivity analysis. Moreover, sensitivity analysis of weak efficiency is given in [27]. 
Here, we are going to use the following approach to sensitivity analysis: as decision maker chooses parameter $q *$ he or she wants to know for which values of $q$ the obtained solution is still the compromise solution. Thus, we proceed sensitivity analysis of the compromise solution by means of the values of $q$. In other words we solve problem (2.2) with the given parameter $p *$ and parameters $q$ from the neighborhood of the given parameter $q *$ and check if a given compromise solution is optimal for (2.2). In order to solve this problem, simulation techniques are used. These methods consists of review parameters $q$ and solve problem (2.2) with $p^{*}$ and chosen $q$.

The similar procedure of sensitivity analysis is used also for parameter $p *$. It means that we solve problem (2.2) with the given parameter $q *$ and parameters $p$ from the neighborhood of the given parameter $p *$ and check if a given compromise solution is still optimal for problem (2.2).

\subsection{ICOH METHOD}

On the basis of the previous sections we can formulate interactive compromise hypersphere $(\mathrm{ICOH})$ method. The $\mathrm{ICOH}$ method consist of the following steps:

ICOH 1. Decision maker chooses the finite set of nondominated solutions for the given multi-objective problem (MOP).

ICOH 2. Decision maker chooses the parameter $q$ as the representation of his risk aversion following the rule: the greater risk aversion the greater $q$. We obtain parameter $q^{*}$ (see Sect. 4.3).

ICOH 3. Solve problem (4.1) with fixed parameter $q^{*}$ and some parameters $p$ and obtain parameter $p^{*}$ (see Sect. 4.1). We propose to review three most popular parameters $p=1,2, \infty$.

ICOH 4. Choose the compromise solution $\overline{\mathbf{y}^{i}}$ for compromise hypersphere method with $p^{*}, q^{*}$ (see Sect. 2.2)

ICOH 5. Sensitivity analysis of the found compromise solution by means of changing parameters $q$ and $p$ (see Sect. 4.4).

\section{Applications and numerical examples of ICOH method}

\subsection{Assignment Problem}

We consider the multi-criteria assignment problem. In this problem we have a number of persons and a number of activities. Any person can be assigned to any activity, incurring some criteria (for example cost, time, distance). It is required to perform all activities by assigning exactly one person to each activity in such a way that the criteria are minimized. Methods of computing the nondominated solutions of the problem are considered in many papers, see for example: [31,33,35]. Let us consider a bi-criteria example from work by White [35]. To make this example more interesting, we extend it by adding one person and one activity. The considered 
multi-objective programming problem has the following form:

$$
\begin{gathered}
v \min \left[\begin{array}{c}
3 x_{11}+2 x_{12}+x_{13}+2 x_{21}+3 x_{22}+100 x_{23} \\
+x_{24}+x_{31}+100 x_{32}+3 x_{23}+x_{41}+x_{42} \\
x_{11}+4 x_{12}+3 x_{13}+4 x_{14}+x_{21}+x_{22} \\
+100 x_{23}+3 x_{31}+100 x_{32}+x_{33}+2 x_{34}+3 x_{42}
\end{array}\right] \\
\quad \sum_{j=1}^{4} x_{i j}=1, \quad i=1, \ldots, 4 \\
\sum_{i=1}^{4} x_{i j}=1, \quad j=1, \ldots, 4 \\
x_{i j} \in\{0,1\}, \quad i, j=1, \ldots, 4 .
\end{gathered}
$$

We will conduct the $\mathrm{ICOH}$ method described in Section 4.5.

ICOH 1. Decision maker chooses all nondominated solutions of the problem (5.1):

$$
\mathbf{y}^{1}=[4,7], \quad \mathbf{y}^{2}=[5,6], \quad \mathbf{y}^{3}=[6,4], \quad \mathbf{y}^{4}=[9,3] .
$$

The solutions can be obtained by using methods presented in [35].

ICOH 2. Decision maker chooses the parameter $q^{*}=\infty$ as the representation of his extremely high risk aversion.

ICOH 3. For $q^{*}=\infty$. and $p=1,2, \infty$ we solve problem (4.1) and obtain the following results:

$$
\begin{aligned}
\min \left\{\overline{\min }(2.2)^{1, \infty}, \overline{\min }(2.2)^{2, \infty}, \overline{\min }(2.2)^{\infty, \infty}\right\} & =\min \{0.500,0.264,0.502\} \\
& =0.264=\overline{\min }(2.2)^{2, \infty}
\end{aligned}
$$

It means that $p=2$ suits better than $p=1$ and $p=\infty$. Thus we choose $p^{*}=2$.

ICOH 4. On the basis of the distance to the previously found hypersphere we obtain the following ranking.

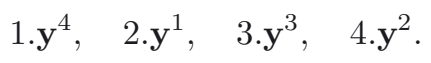

Thus we have $\mathbf{y}^{4}$ as the compromise solution.

ICOH 5. We obtain the that $\mathbf{y}^{4}$ is the compromise solution for all $q \in[1, \infty)$ and $p^{*}=2$. It means that the compromise solution $\mathbf{y}^{4}$ is not sensitive to changing the parameter $q^{*}$. Furthermore, we obtain the that $\mathbf{y}^{4}$ is the compromise solution for all $p \in[1,3]$ and $q^{*}=\infty$. It means that the compromise solution $\mathbf{y}^{4}$ is sensitive to changing the parameter $p^{*}$. 


\subsection{KNAPSACK PROBLEM}

We consider the multicriteria knapsack problem. In this problem we have a set of items, each with a weight and multi-values (multi-criteria optimized). We determine the number of each item to include in a collection so that the total weight is less than or equal to a given limit and the given values (criteria) are maximized. Methods of computing the nondominated solutions of the multi-criteria knapsack problem are considered in papers by $[3,6,11]$. Let us consider a bi-criteria knapsack problem from [16]. The considered multi-objective programming problem has the following form:

$$
\begin{gathered}
v \max \left[\begin{array}{c}
85 x_{1}+31 x_{2}+33 x_{3}+25 x_{4}+28 x_{5}+15 x_{6}+29 x_{7} \\
72 x_{1}+17 x_{2}+47 x_{3}+83 x_{4}+49 x_{5}+88 x_{6}+78 x_{7}
\end{array}\right] \\
98 x_{1}+74 x_{2}+94 x_{3}+91 x_{4}+51 x_{5}+57 x_{6}+57 x_{7} \leq 261 \\
x_{i} \in\{0,1\}, \quad i=1, \ldots, 7
\end{gathered}
$$

We will use the ICOH method described in Section 4.5.

ICOH 1. Decision maker chooses all nondominated solutions of the prob$\operatorname{lem}(5.2)$ :

$$
\begin{aligned}
& \mathbf{y}^{1}=[97,298], \mathbf{y}^{2}=[139,233], \mathbf{y}^{3}=[147,197], y^{4}=[105,262] \\
& \mathbf{y}^{5}=[125,243], \mathbf{y}^{6}=[129,238], \mathbf{y}^{7}=[142,199] .
\end{aligned}
$$

The above nondominated solutions can be obtained by using methods presented in [16].

ICOH 2. Decision maker chooses the parameter $q^{*}=1$ as the representation of his extremely low risk aversion.

ICOH 3. For $q^{*}=1$. and $p=1,2, \infty$ we solve problem (4.1) and obtain the following results:

$$
\begin{aligned}
\min \left\{\overline{\min }(2.2)^{1, \infty}, \overline{\min }(2.2)^{2, \infty}, \overline{\min }(2.2)^{\infty, \infty}\right\} & =\min \{40.02,24.296,99.70\} \\
& =24.296=\overline{\min }(2.2)^{2, \infty}
\end{aligned}
$$

It means that $p=2$ suits better than $p=1$ and $p=\infty$. Thus we choose $p^{*}=2$.

ICOH 4. On the basis of the distance to the previously found hypersphere we obtain the following ranking.

$$
1 . \mathbf{y}^{1}, 2 \cdot \mathbf{y}^{5}, 3 \cdot \mathbf{y}^{6}, 4 \cdot \mathbf{y}^{3}, 5 \cdot \mathbf{y}^{2}, 6 \cdot \mathbf{y}^{4}, 7 \cdot \mathbf{y}^{7} .
$$

Thus we have $\mathbf{y}^{1}$ as the compromise solution.

ICOH 5. We obtain the that $\mathbf{y}^{1}$ is the compromise solution for $q \in[1,1.755]$ and $p^{*}=2$. It means that the compromise solution $\mathbf{y}^{1}$ is very sensitive to changing the parameter $q^{*}$. Furthermore, we obtain the that $\mathbf{y}^{1}$ is the compromise solution for all $p \in[1,6.5]$ and $q^{*}=1$. It means that the compromise solution $\mathbf{y}^{1}$ is not sensitive to changing the parameter $p^{*}$. 
TABLE 2. Data of the project management.

\begin{tabular}{|l|c|c|c|c|}
\hline $\begin{array}{l}\text { Activity } \\
\text { with Description }\end{array}$ & Depends on & $\begin{array}{c}\text { Normal time } \\
\text { (days) }\end{array}$ & $\begin{array}{c}\text { Crash cost } \\
\text { per day }\end{array}$ & $\begin{array}{c}\text { Maximal } \\
\text { crashed days }\end{array}$ \\
\hline $\begin{array}{l}\text { A - Prepare technical } \\
\text { specifications }\end{array}$ & - & 10 & 5.5 & 2 \\
\hline B- Tender Processing & A & 25 & 30 & 1 \\
\hline C- Release of work orders & B & 3 & 13 & 1 \\
\hline $\begin{array}{l}\text { D- Supply of boiler } \\
\text { equipment }\end{array}$ & $\mathrm{C}$ & 60 & 7 & 2 \\
\hline E- Supply of auxiliaries & $\mathrm{C}$ & 20 & 10 & 1 \\
\hline $\begin{array}{l}\text { F- Supply of pipes } \\
\text { \& pipe fittings }\end{array}$ & $\mathrm{C}$ & 10 & 2.2 & 1 \\
\hline G- Civil work & $\mathrm{C}$ & 15 & 10 & 2 \\
\hline $\begin{array}{l}\text { H- Installation of auxiliary } \\
\text { equipment and piping }\end{array}$ & $\mathrm{E}, \mathrm{F}, \mathrm{G}$ & 5 & 10 & 1 \\
\hline I- Installation of boiler & $\mathrm{D}, \mathrm{H}$ & 10 & 10 & 1 \\
\hline $\begin{array}{l}\text { J- Testing and } \\
\text { commissioning }\end{array}$ & $\mathrm{I}$ & 2 & 10 & 1 \\
\hline
\end{tabular}

\subsection{Project management problem}

We consider the project management problems by means of time-cost analysis. General models of project management are presented in [19]. The time-cost analysis is a generalization of CPM method (critical Path method) a well-known approach to the project managements, (CPM approach is given for example in [36]). Let us consider a management project from work by Elmabrouk in [8]. Table 2 shows a list of various activities needed for replacing an existing boiler with an energy efficient boiler. This table also presents the rest of data used in the time-cost analysis in project management. We take into account two criteria:

- minimizing the time of finishing the project;

- minimizing the crash cost.

The MOLP problem built for the project from Table 2 has the following form:

$v \min \left[\begin{array}{c}5.5 x_{A}+30 x_{B}+13 x_{C}+7 x_{D}+10 x_{E}+2.2 x_{F}+10 x_{G}+10 x_{H}+10 x_{I}+10 x_{J} \\ y_{\text {finish }}\end{array}\right]$

$y_{B}+x_{A} \geq 10, y_{C}-y_{B}+x_{B} \geq 25, \quad y_{D}-y_{C}+x_{C} \geq 3, \quad y_{E}-y_{C}+x_{C} \geq 3$,

$y_{F}-y_{C}+x_{C} \geq 3, y_{G}-y_{C}+x_{C} \geq 3, \quad y_{H}-y_{G}+x_{G} \geq 15, \quad y_{H}-y_{F}+x_{F} \geq 10$,

$y_{H}-y_{E}+x_{E} \geq 20, \quad y_{I}-y_{H}+x_{H} \geq 5, \quad y_{I}-y_{D}+x_{D} \geq 60, \quad y_{J}-y_{I}+x_{I} \geq 10$,

$y_{\text {finish }}-y_{J}+x_{J} \geq 2$,

$0 \leq x_{A} \leq 2, \quad 0 \leq x_{B} \leq 1, \quad 0 \leq x_{C} \leq 1, \quad 0 \leq x_{D} \leq 2, \quad 0 \leq x_{E} \leq 1$,

$0 \leq x_{F} \leq 1, \quad 0 \leq x_{G} \leq 2, \quad 0 \leq x_{H} \leq 1, \quad 0 \leq x_{I} \leq 1, \quad 0 \leq x_{J} \leq 1$,

$y_{B}, y_{C}, y_{D}, y_{E}, y_{F}, y_{G}, y_{H}, y_{I}, y_{J} \geq 0$. 
We will use the ICOH conduct described in Section 4.5.

ICOH 1. Decision maker chooses all nondominated solutions of problem (5.3):

$$
\begin{aligned}
& \mathbf{y}^{1}=[88,102], \mathbf{y}^{2}=[58,103], \mathbf{y}^{3}=[45,104], \mathbf{y}^{4}=[35,105], \mathbf{y}^{5}=[25,106] \\
& \mathbf{y}^{6}=[0,110] .
\end{aligned}
$$

ICOH 2. Decision maker chooses the parameter $q^{*}=2$ as the representation of his low risk aversion.

ICOH 3. For $q^{*}=2$. and $p=1,2, \infty$ we solve problem (4.1) and obtain an optimal solution $p^{*}=2$.

ICOH 4. On the basis of the distance to the previously found hypersphere we obtain $\mathbf{y}^{4}$ as the compromise solution.

ICOH 5. We obtain the that $\mathbf{y}^{4}$ is the compromise solution for $q \in[1.422,2.814]$ and $p^{*}=2$. It means that the compromise solution $\mathbf{y}^{4}$ is sensitive to changing the parameter $q^{*}=1$. Furthermore, we obtain the that $\mathbf{y}^{4}$ is the compromise solution for all $p \in[1,2.563]$ and $q^{*}=2$. It means that the compromise solution $\mathbf{y}^{4}$ is sensitive to changing the parameter $p^{*}$.

\subsection{Manufacturing Problem (ACtivity-Analysis Problem)}

We consider a well-known problem of linear programming - the manufacturing problem. This problem is analysed in case of one-criterion for example in works $[12,13]$ by Gass. We extend the one-criterion manufacturing problem to multi-objective problem in the following way. By using the initial description of Gass [13] we formulate the following problem. A manufacturing company has at its disposal fixed amounts of different resources. These resources, such as raw material, labour etc. can be combined to produce any one of several different commodities. The company knows how much of resources should be taken to produce commodities. It also knows how much profit it makes for each commodity. The company desires to produce the combination of commodities with multiple criteria:

- maximizing the total profit;

- minimizing the usage of given deficient resources.

As a numerical example we use an example from work [13]. A bakery starts the day with a certain supply of flour, shortening, eggs, milk, and yeast. It specializes in making four types of cakes (we assume that these products can be made in arbitrary amount of weights - pounds). Furthermore, it has to make at least 50 pounds of each cakes. We consider two criteria: maximizing the profit and minimizing the usage of flour. The recipes are given in Table 3 (we ignore such plentiful supplies as salt, water, etc.). 
TABLE 3. Data for the manufacturing problem.

\begin{tabular}{|c|c|c|c|c|c|}
\hline & cake 1 & cake 2 & cake 3 & cake 4 & available resources \\
\hline shortening & 2 & 12 & 3 & 4 & 4000 \\
\hline eggs & 0 & 3 & 3 & 1 & 3000 \\
\hline sugar & 0.25 & 1.5 & 0.125 & 1 & 500 \\
\hline milk & 2 & 0.75 & 1 & 0 & 2000 \\
\hline yeast & 1 & 0 & 1 & 0 & 1000 \\
\hline flour & 12 & 3 & 4.5 & 1.5 & MIN \\
\hline profit & 2 & 3 & 4 & 2 & MAX \\
\hline
\end{tabular}

The above problem can be formulated in MOLP form. The description is as follows:

$$
\begin{array}{r}
v \max \left[\begin{array}{c}
2 x_{1}+3 x_{2}+4 x_{3}+2 x_{4} \\
-12 x_{1}-3 x_{2}-4.5 x_{3}-1.5 x_{4}
\end{array}\right] \\
2 x_{1}+12 x_{2}+3 x_{3}+4 x_{4} \leq 4000 \\
3 x_{2}+3 x_{3}+x_{4} \leq 3000 \\
0.25 x_{1}+1.5 x_{2}+0.125 x_{3}+x_{4} \leq 500 \\
2 x_{1}+0.75 .5 x_{2}+x_{3} \leq 2000 \\
x_{1}+x_{3} \leq 1000 x_{1}, x_{2}, x_{3}, x_{4} \geq 50 .
\end{array}
$$

We will use the ICOH method described in Section 4.5.

ICOH 1. Decision maker chooses all nondominated solutions of problem (5.4):

$$
\begin{aligned}
& \mathbf{y}^{1}=[575,1050], \mathbf{y}^{2}=[1287.5,1584.4], \mathbf{y}^{3}=[3575,4215], \mathbf{y}^{4}=[4175,5025], \\
& \mathbf{y}^{5}=[4259.1,5515.9]
\end{aligned}
$$

ICOH 2. Decision maker chooses the parameter $q^{*}=1$ as the representation of his extremely low risk aversion.

ICOH 3. For $q^{*}=1$. and $p=1,2, \infty$ we solve problem (4.1) and obtain an optimal solution $p^{*}=2$.

ICOH 4. On the basis of the distance to the previously found hypersphere we obtain $\mathbf{y}^{2}$ as the compromise solution.

ICOH 5. We obtain the that $\mathbf{y}^{2}$ is the compromise solution for $q \in[1, \infty]$ and $p^{*}=2$. It means that the compromise solution $\mathbf{y}^{2}$ is not sensitive to changing the parameter $q^{*}$. Furthermore we obtain the that $\mathbf{y}^{2}$ is the compromise solution for all $p \in[1,3.1]$ and $q^{*}=1$. It means that the compromise solution $\mathbf{y}^{2}$ is sensitive to changing the parameter $p^{*}$.

\section{Conclusions And FURTher RESEARCH}

The paper focused on the decision support in the MOLP problems. We have presented a method to ranking of the chosen nondominated solutions. The presented method is based on finding a hypersphere which is closest to the set of the chosen solutions. The method proposed by Gass and Roy in [14] has been 
developed by using different ways of measuring the distance. The motivation to study other metrics was to give the decision maker more opportunities to choose the metric and in this way show his risk aversion. The presented analysis gave some remarks how to choose the proper metric. Moreover, we presented the interactive version of the method to include the decision maker preferences - the $\mathrm{ICOH}$ method with sensitivity analysis. This method is a decision tool in multiple objective linear programming problems. The aim of the $\mathrm{ICOH}$ method is to choose a compromise solution, which is based on choosing proper metrics by the decision maker. We presented applications of the $\mathrm{ICOH}$ method in the form of the assignment problem, the knapsack problem, the project management problem and the manufacturing problem. Further research and problems to solve, according to the author, are as follows:

- numerical analysis of algorithms searching for the optimal solutions of problem (2.2) by using various optimization methods: [22,24];

- extension using the augmented Tchebycheff metric: [29];

- comparison with outranking methods based on compromise programming: [20, 21];

- including more general structure to describe the preferences of decision maker like fuzzy numbers or stochastic dominance: [26,32].

\section{REFERENCES}

[1] G.T. Anthony, B. Bittner, B.P. Butler, M.G. Cox, R. Elligsen, A.B. Forbes, H. Gross, S.A. Hannaby, P.M. Harris and J. Kok, Chebychev reference software for evaluation of coordinate measuring machine data, Report EUR 15304 EN, National Physical Laboratory, Teddington, United Kingdom (1993).

[2] E. Ballestero, Selecting the CP metric: A risk aversion approach. Eur. J. Oper. Res. 97 (1996) 593-596.

[3] C. Bazgan, H. Hugot and D. Vanedrpooten, Solving efficiently the 0-1 multi-objective knapsack problem. Comput. Oper. Res. 36 (2009) 260-279.

[4] B.P. Butler, A.B. Forbes and P.M. Harris, Algorithms for geometric tolerance assessment. NPL Report DITC 228/94, National Physical Laboratory, Teddington, United Kingdom (1994).

[5] E. Carrizosa, E. Conde, A. Pacual, D. Romero-Morales, Closest solutions in ideal-point methods, in Advances in multiple objective and goal programming, edited by R. Caballero, F. Ruiz and R.E. Steuer. Springer Verlag, Berlin, LNEMS 455 (1996) 274-281.

[6] M. Eben-Chaime, Parametric solution for linear bicriteria knapsack models. Manag. Sci. 42 (1996) 1565-1575.

[7] M. Ehrgott, Multicriteria optimization. Springer Verlag, Berlin (2002).

[8] O.M. Elmabrouk, A linear programming technique for the pptimization of the activities in maintenance projects. Int. J. Eng. Technol. IJET-IJENS 11 (2011).

[9] J.P. Evans and R.E. Steuer, A revised simplex method for linear multiple objective programs. Math. Programm. 5 (1973) 54-72.

[10] T. Gal and K. Wolf, Stability in vector maximization - a survey. Eur. J. Oper. Res. 25 (1986) 169-182.

[11] X. Gandibleux and A. Freville, Tabu search based procedure for solving the 0/1 multiobjective knapsack problem: The two objective case. J. Heuristics 6 (2000) 361-383.

[12] S.I. Gass, An illustrated guide to linear programming. Mc Graw-Hill Book Company, New York (1970). 
[13] S.I. Gass, Linear programming, methods and applications. Mc Graw-Hill Book Company, New York (1975).

[14] S.I. Gass and P.G. Roy, The compromise hypersphere for multiobjective linear programming. Eur. J. Oper. Res. 144 (2003) 459-479.

[15] S.I. Gass, H.H. Harary and C. Witzgall, Fitting circles and spheres to coordinate measuring machine data. Int. J. Flexible Manuf. 10 (1998) 5-25.

[16] C. Gomes da Silva, J. Climaco and J. Rui Figueria, Core problem in bi-criteria $\{0,1\}$ knapsack problem. Comput. Oper. Res. 35 (2008) 2292-2306.

[17] P. Hansen, M. Labbe and R.E. Wendell, Sensitivity analysis in multiple objective linear programming: the tolerance approach. Eur. J. Oper. Res. 38 (1989) 63-69.

[18] M. Hladik, Additive and multiplicative tolerance in multiobjective linear programming. Oper. Res. Lett. 36 (2008) 393-396.

[19] J. Knutson and I. Bitz, Project Management. Amacom, New York (1991).

[20] S. Opricovic and G.H. Tzeng, Compromise solution by MCDM methods: a comparative analysis of VIKOR and TOPSIS. Eur. J. Oper. Res. 156 (2004) 445-455.

[21] S. Opricovic and G.H. Tzeng, Extended VIKOR method in comparison with outranking methods. Eur. J. Oper. Res. 178 (2007) 514-529.

[22] S. Sitarz, Hybrid methods in multi-criteria dynamic programming. Appl. Math. Comput. 180 (2006) 38-45.

[23] S. Sitarz, Postoptimal analysis in multicriteria linear programming. Eur. J. Oper. Res. 191 (2008) 7-18.

[24] S. Sitarz, Ant algorithms and simulated annealing for multicriteria dynamic programming. Comput. Oper. Res. 36 (2009) 433-441.

[25] S. Sitarz, Standard sensitivity analysis and additive tolerance approach in MOLP. Ann. Oper. Res. 181 (2010) 219-232.

[26] S. Sitarz, Dynamic programming with ordered structures: theory, examples and applications. Fuzzy Sets and Systems 161 (2010) 2623-2641.

[27] S. Sitarz, Sensitivity analysis of weak efficiency in MOLP. Asia-Pacific J. Oper. Res. 28 (2011) 445-455.

[28] S. Sitarz, Mean value and volume-based sensitivity analysis for Olympic rankings. Eur. J. Oper. Res. 216 (2012) 232-238.

[29] R.E. Steuer and E. Choo An Interactive weighted Tchebycheff procedure for multiple objective programming. Math. Programm. 26 (1981) 326-344.

[30] R. Steuer, Multiple criteria optimization theory: computation and application. John Willey, New York (1986).

[31] D. Tuyttens, J. Teghem, P. Fortemps and K. Van Nieuwenhuyse, Performance of the MOSA method for the bicriteria assignment problem. J. Heuristics 6 (2000) 259-310.

[32] T. Trzaskalik and S. Sitarz, Discrete dynamic programming with outcomes in random variable structures. Eur. J. Oper. Res. 177 (2007) 1535-1548.

[33] E.L. Ulungu and J. Teghem, Multicriteria assignment problem - a new method. Technical Report, Faculte Polytechnique de Mons, Belgium (1992).

[34] A.P. Wierzbicki, Reference point approaches, in Multicriteria Decision Making, edited by R. Gal, T.J. Stewart, T. Hanne. Kluwer, Boston, MA (Chap. 9) (1999).

[35] D.J. White, A special Multi-objective assigmnet problem. J. Oper. Res. Soc. 35 (1984) 759-767.

[36] A. Woolf and B. Murray, Faster construction projects with CPM Scheduling. McGraw Hill (2007).

[37] P.L. Yu and M. Zeleny, The set of all nondominated solutions in linear cases and a multicriteria simplex method. J. Math. Anal. Appl. 49 (1975) 430-468.

[38] M. Zeleny, Multiple Criteria Decision Making. McGraw-Hill Book Company, New York (1982).

[39] S. Zionts and J. Wallenius, An interactive multiple objective linear programming method for a class of underlying nonlinear utility functions. Manag. Sci. 29 (1983) 519-529. 